\title{
HOMOCLINIC ORBITS AND CRITICAL POINTS OF BARRIER FUNCTIONS
}

\author{
PIERMARCO CANNARSA AND WEI CHENG
}

\begin{abstract}
We interpret the close link between the critical points of Mather's barrier functions and minimal homoclinic orbits with respect to the Aubry sets on $\mathbb{T}^{n}$. We also prove a critical point theorem for barrier functions, and the existence of such homoclinic orbits on $\mathbb{T}^{2}$ as an application.
\end{abstract}

\section{INTRODUCTION}

In the huge literature that is devoted to the study of homoclinic orbits of Hamiltonian systems and dates back, at least, to the works of Poincaré, one can single out one important approach which is based on the Ambrosetti-Rabinowitz critical point theory see, e.g., 9, $, 8,[10,32$. Another powerful viewpoint in this context is provided by Mather's theory [28, 29] and weak KAM theory (see, e.g. Fathi's book 21]) which helped to clarify many aspects of minimal orbits and invariant sets, see, e.g., [20, [4, ,17, ,19, 34, 335.

In this paper, we adopt a mixed strategy to investigate the existence of minimal homoclinic orbits with respect to the Aubry sets for a given Tonelli Hamiltonian, using critical point theory for certain barrier functions. We concentrate on the case of the $n$-torus throughout the paper, even if some of our results can be proved for more general manifolds using similar ideas.

Let $H$ be a Tonelli Hamiltonian on $\mathbb{T}^{n}$, and consider the associated HamiltonJacobi equation which has the form

$$
H(x, c+D u(x))=\alpha(c), \quad x \in \mathbb{T}^{n},
$$

where $c \in \mathbb{R}^{n}$ stands for a cohomology class in $H^{1}\left(\mathbb{T}^{n}, \mathbb{R}\right)$ and $\alpha(\cdot)$ is Mather's function. For fixed $c$, under the generic condition that the Aubry class is unique, it is easy to define the barrier function $B_{c}^{*}$ as difference of two weak KAM solutions forming a conjugate pair $\left(u_{c}^{-}, u_{c}^{+}\right)$, that is,

$$
B_{c}^{*}(x)=u_{c}^{-}(x)-u_{c}^{+}(x), \quad x \in \mathbb{T}^{n} .
$$

As is well-known for viscosity solutions, $B_{c}^{*}$ turns out to be a locally semiconcave function with linear modulus, see [13. Moreover, in [12, it was proved that $x$ determines a homoclinic orbit with respect to the projected Aubry set $\mathscr{A}_{c}$ whenever $x$ is a critical point of $B_{c}^{*}$ outside $\mathscr{A}_{c}$ and the limiting differentials $D^{*} u_{c}^{-}(x)$ and $D^{*} u_{c}^{+}(x)$ have a nonempty intersection. More precisely, one can show that there

Date: September 25, 2018.

2010 Mathematics Subject Classification. 26B25, 35A21, 49L25, 37J50, 70H20.

Key words and phrases. Semiconcave functions, Hamilton-Jacobi equations, weak KAM theory, homoclinic orbits. 
exists a $C^{2}$ extremal curve $\gamma:(-\infty, \infty) \rightarrow \mathbb{T}^{n}$ such that $\gamma(0)=x$ and the $\alpha$ - and $\omega$ - limit sets of $\gamma$ belong to $\mathscr{A}_{c}$ even if $x$ is a singular critical point of $B_{c}^{*}$.

Building on the above result, our construction of minimal homoclinic (even heteroclinic) orbits is obtained in two steps:

(1) we need find enough critical points outside $\mathscr{A}_{c}$, and

(2) we need a criterion to ensure that such critical points can indeed create minimal homoclinic orbits.

For the first step, we prove the following.

Theorem 1.1. Let $L$ be a Tonelli Lagrangian on $\mathbb{T}^{n}$, and for fixed $c \in \mathbb{R}^{n}$, suppose the projected Aubry set $\mathscr{A}_{c}$ consists of a single Aubry class. Then there exist at least $\operatorname{Cat}\left(\mathbb{T}^{n} \backslash U\right)$ critical points of the barrier function $B_{c}^{*}$ outside $\mathscr{A}_{c}$, where $U$ is any open neighborhood of $\mathscr{A}_{c}$.

In the above statement Cat $\left(\mathbb{T}^{n} \backslash U\right)$ stands for the Lusternik-Schnirelmann category of $\mathbb{T}^{n} \backslash U$. Moreover, we recall that the uniqueness of the Aubry class holds for a generic Tonelli Hamiltonian as explained in Section 2 below.

For the second step, we give the following criterion where $\Lambda_{x}^{+}$denotes the superlevel set of $B_{c}^{*}$ at $x$.

Theorem 1.2. Let $x \in \mathbb{R}^{n}$ be a critical point of $B_{c}^{*}$. Then we have that

$$
0 \in D^{*} u_{c}^{-}(x)-D^{*} u_{c}^{+}(x)
$$

if any of the following conditions is satisfied:

(a) at least one of the two solutions $v^{-}$and $v^{+}$is differentiable at $x$,

(b) the tangent space to $\Lambda_{x}^{+}$at $x$ is such that

$$
\operatorname{dim}\left(\operatorname{Tan}\left(x, \Lambda_{x}^{+}\right)\right) \geqslant n-1,
$$

(c) $n=2$ and there exists a unit vector $\theta$ such that $\langle p, \theta\rangle \geqslant 0$ for all $p \in D^{+} B_{c}^{*}(x)$,

(d) $n=2$ and $x$ is not an isolated critical point of $B_{c}^{*}$.

Since a $B_{c}^{*}$ is at most semiconcave, critical points have to be interpreted and dealt with in a nonsmooth setting. For this purpose, we borrow a result from Attouch [3] which applies the Lasry-Lions regularization method to critical points, see Proposition 3.5 below. Appealing to the above theorems, we obtain the following.

Theorem 1.3. Let $L$ be a Tonelli Lagrangian on $\mathbb{T}^{2}$, and for fixed $c \in \mathbb{R}^{2}$, suppose the projected Aubry set $\mathscr{A}_{c}$ consists of a single Aubry class. If there exists an open neighborhood $U \subset \mathbb{T}^{2}$ of $\mathscr{A}_{c}$ such that $\mathbb{T}^{2} \backslash U$ is non-contractible, then there exists a minimal homoclinic orbit with respect to the Aubry set, $\tilde{\mathscr{A}}_{c}$, lying outside $\tilde{\mathscr{A}}_{c}$.

Is is worth noting that our approach also works to construct connecting orbits among distinct Aubry classes if the projected Aubry set has more than one class.

Theorem 1.4. Let $L$ be a Tonelli Lagrangian on $\mathbb{T}^{2}$, and for fixed $c \in \mathbb{R}^{2}$, suppose the projected Aubry set $\mathscr{A}_{c}$ consists of finitely many Aubry classes. If there exists an open neighborhood $U \subset \mathbb{T}^{2}$ of $\mathscr{A}_{c}$ such that $\mathbb{T}^{2} \backslash U$ is non-contractible, then there must be a connecting orbit between any pair of distinct Aubry classes.

The paper is organized as follows. In Section 2, we recall basic material on semiconcave functions and weak KAM theory. In section 3, we discuss the homoclinic phenomenon and critical points of semiconcave functions, and prove the main results of the paper. 
Acknowledgments This work was partially supported by the Natural Scientific Foundation of China (Grant No. 11271182), the National Basic Research Program of China (Grant No. 2013CB834100), and the National Group for Mathematical Analysis and Probability of the Italian Istituto Nazionale di Alta Matematica "Francesco Severi". The authors are grateful to Albert Fathi, Ludovic Rifford and Antonio Siconolfi for helpful discussions and comments on the results of this paper.

\section{Preliminary facts}

2.1. Hamilton-Jacobi equations and viscosity solutions. Let $\mathbb{T}^{n}$ be the $n$ dimensional torus. We denote by $T \mathbb{T}^{n}$ the tangent bundle of $\mathbb{T}^{n}$ and by $T^{*} \mathbb{T}^{n}$ the cotangent bundle.

Definition 2.1. A function $L: T \mathbb{T}^{n} \rightarrow \mathbb{R}$ is said to be a Tonelli Lagrangian if the following assumptions are satisfied.

(L1) Smoothness: $L=L(x, v)$ is of class at least $C^{2}$.

(L2) Convexity: The Hessian $\frac{\partial^{2} L}{\partial v^{2}}(x, v)$ is positive definite on each fibre $T_{x} \mathbb{T}^{n}$.

(L3) Superlinearity:

$$
\lim _{|v| \rightarrow \infty} \frac{L(x, v)}{|v|}=\infty \quad \text { uniformly for } x \in \mathbb{T}^{n} .
$$

Given a Tonelli Lagrangian $L$, the Tonelli Hamiltonian $H=H(x, p)$ associated with $L$ is defined as follows:

$$
H(x, p)=\max \left\{\langle p, v\rangle-L(x, v): v \in T_{x} \mathbb{T}^{n}\right\}, \quad(x, p) \in T^{*} \mathbb{T}^{n} .
$$

It is easy to see that for any Tonelli Lagrangian $L$, the associated Hamiltonian $H$ satisfies similar smoothness ( $H$ is of class at least $C^{2}$ ), convexity, and superlinearity conditions, which will be referred to as (H1), (H2), and (H3).

Throughout this paper we will be concerned with the Hamilton-Jacobi equation

$$
H_{c}(x, D u(x))=H(x, c+D u(x))=\alpha(c) \quad\left(x \in \mathbb{T}^{n}\right)
$$

with $H$ any Tonelli Hamiltonian and $H_{c}(x, \cdot):=H(x, c+\cdot)$, where $c \in \mathbb{R}^{n}$ and $\alpha$ : $\mathbb{R}^{n} \rightarrow \mathbb{R}$ is Mather's $\alpha$-function. In other words, we can suppose that $H=H(x, p)$ is $\mathbb{Z}^{n}$-periodic in the $x$ variable, convex and superlinear in the $p$ variable, and $u$ is a $\mathbb{Z}^{n}$-periodic solution of (2.1).

We say that $u: \mathbb{T}^{n} \rightarrow \mathbb{R}$ is a viscosity subsolution (resp. supersolution) of (2.1), if for each $C^{1}$ function $\phi: M \rightarrow \mathbb{R}$ such that $u-\phi$ admits a maximum (resp. a minimum) at $x \in M$, we have

$$
H_{c}(x, D \phi(x)) \leqslant \alpha(c), \quad\left(\operatorname{resp} . H_{c}(x, D u(x)) \geqslant \alpha(c)\right) .
$$

We say that $u: \mathbb{T}^{n} \rightarrow \mathbb{R}$ is a viscosity solution, if it is both a subsolution and a supersolution. A viscosity solution of (2.1) is called a critical viscosity solution.

2.2. Semiconcave functions. Let $\Omega \subset \mathbb{R}^{n}$ be open and convex. A function $u$ : $\Omega \rightarrow \mathbb{R}$ is semiconcave (with linear modulus) if there exists a constant $C>0$, such that

$$
\lambda u(x)+(1-\lambda) u(y)-u(\lambda x+(1-\lambda) y) \leqslant \frac{C}{2} \lambda(1-\lambda)|x-y|^{2}
$$

for any $x, y \in \Omega$ and $\lambda \in[0,1]$, and $C$ is called a semiconcavity constant for $u$ in $\Omega$. A function $u: \Omega \rightarrow \mathbb{R}$ is said to be locally semiconcave if for any $x \in \Omega$, there exists an open convex subset $U \ni x$ such that $\left.u\right|_{U}$ is semiconcave. 
Let $u: \Omega \subset \mathbb{R}^{n} \rightarrow \mathbb{R}$ be a semiconcave function, for any $x \in \Omega$, the set

$$
\begin{aligned}
& D^{-} u(x)=\left\{p \in \mathbb{R}^{n}: \liminf _{y \rightarrow x} \frac{u(y)-u(x)-\langle p, y-x\rangle}{|y-x|} \geqslant 0\right\}, \\
& D^{+} u(x)=\left\{p \in \mathbb{R}^{n}: \limsup _{y \rightarrow x} \frac{u(y)-u(x)-\langle p, y-x\rangle}{|y-x|} \leqslant 0\right\} .
\end{aligned}
$$

are called the (Dini) subdifferential and superdifferential of $u$ at $x$ respectively.

Proposition $2.2(13)$. Let $u: \Omega \rightarrow \mathbb{R}$ be a function on $\Omega \subset \mathbb{R}^{n}$. If there exists a constant $C>0$ such that, for any $x \in \Omega$, there exists $p \in \mathbb{R}^{n}$ such that

$$
u(y) \leqslant u(x)+\langle p, y-x\rangle+\frac{C}{2}|y-x|^{2}, \quad \forall y \in \Omega,
$$

then $u$ is semiconcave with constant $C$.

If $u$ is semiconcave function on $\Omega \subset \mathbb{R}^{n}$ with constant $C$, then (2.2) holds for any $p \in D^{+} u(x)$.

Let $u: \Omega \rightarrow \mathbb{R}$ be locally Lipschitz. A vector $p \in \mathbb{R}^{n}$ is called a limiting differential of $u$ at $x$ if there exists a sequence $\left\{x_{n}\right\} \subset \Omega \backslash\{x\}$ such that $u$ is differentiable at $x_{k}$ for each $k \in \mathbb{N}$, and

$$
\lim _{k \rightarrow \infty} x_{k}=x \text { and } \lim _{k \rightarrow \infty} D u\left(x_{k}\right)=p .
$$

The set of all limiting differentials of $u$ at $x$ is denoted by $D^{*} u(x)$.

Proposition 2.3. Let $u: \Omega \subset \mathbb{R}^{n} \rightarrow \mathbb{R}$ be a semiconcave function and $x \in \Omega$. Then the following properties hold.

(a) $D^{+} u(x)$ is a nonempty compact convex set in $\mathbb{R}^{n}$ and $D^{*} u(x) \subset \partial D^{+} u(x)$, where $\partial D^{+} u(x)$ denotes the topological boundary of $D^{+} u(x)$.

(b) The set-valued function $x \rightsquigarrow D^{+} u(x)$ is upper semicontinuous.

(c) If $D^{+} u(x)$ is a singleton, then $u$ is differentiable at $x$. Consequently, if $D^{+} u(x)$ is a singleton for every points in $\Omega$, then $u \in C^{1}$.

(d) $D^{+} u(x)$ equals the convex hull of $D^{*} u(x)$.

(e) $D^{*} u(x)=\left\{\lim _{i \rightarrow \infty} p_{i}: p_{i} \in D^{+} u\left(x_{i}\right), x_{i} \rightarrow x, \operatorname{diam}\left(D^{+} u\left(x_{i}\right)\right) \rightarrow 0\right\}$.

A point $x \in \Omega$ is called a singular point of $u$ if $D^{+} u(x)$ is not a singleton. The set of all singular points of $u$, also called the singular set of $u$, is denoted by $\Sigma_{u}$.

2.3. Facts from weak KAM theory. In what follow, $H$ stands for a Tonelli Hamiltonian on the $n$-torus $\mathbb{T}^{n}$ and $L$ for the corresponding Tonelli Lagrangian.

Definition 2.4. Let $L$ be a $C^{2}$ Tonelli Lagrangian on $T \mathbb{T}^{n}$ and set, for any $c \in \mathbb{R}^{n}$,

$$
L_{c}(x, v)=L(x, v)-\langle c, v\rangle \quad \forall(x, v) \in T \mathbb{T}^{n} .
$$

A function $u_{c}: \mathbb{T}^{n} \rightarrow \mathbb{R}$ is said to be dominated by $L_{c}+\alpha(c)$ iff, for each absolutely continuous arc $\gamma:[a, b] \rightarrow \mathbb{T}^{n}$ with $a<b$, one has

$$
u_{c}(\gamma(b))-u_{c}(\gamma(a)) \leqslant \int_{a}^{b} L_{c}(\gamma(s), \dot{\gamma}(s)) d s+\alpha(c)(b-a) .
$$

When this happens, one writes $u_{c} \prec L_{c}+\alpha(c)$. 
Definition 2.5. Let $c \in \mathbb{R}^{n}$, and $u_{c}$ be a real-valued function on $\mathbb{T}^{n}$. An absolutely continuous curve $\gamma:[a, b] \rightarrow \mathbb{T}^{n}$ is said to be $\left(u_{c}, L_{c}, \alpha(c)\right)$-calibrated if

$$
u_{c}(\gamma(b))-u_{c}(\gamma(a))=\int_{a}^{b} L_{c}(\gamma(s), \dot{\gamma}(s)) d s+\alpha(c)(b-a) .
$$

The following well known (see, e.g. 21] 23]) facts are useful to clarify the relation between viscosity solutions and weak KAM solutions.

Proposition 2.6. Let $c \in \mathbb{R}^{n}$. A function $u_{c}: \mathbb{T}^{n} \rightarrow \mathbb{R}$ is dominated by $L_{c}+\alpha(c)$ if and only if $u_{c}$ is a viscosity subsolution of (2.1).

If $u_{c}$ is a viscosity solution of (2.1), then there exists an absolutely continuous arc $\gamma_{x}:(-\infty, 0] \rightarrow \mathbb{T}^{n}$ with $\gamma_{x}(0)=x$ such that $\gamma_{x}$ is $\left(u_{c}, L_{c}, \alpha(c)\right)$-calibrated.

Now, we recall some semiconcavity properties of viscosity solutions. The following result is fundamental (see, e.g., 21] 33).

Proposition 2.7. Any viscosity solution of the Hamilton-Jacobi equation (2.1) is locally semiconcave with linear modulus.

The following is the weak KAM analogue of [13, Theorem 6.4.12]

Proposition 2.8. Ext $D^{+} u(x)=D^{*} u(x)$ for any viscosity solution $u$ of (2.1) and any $x \in \mathbb{T}^{n}$.

Finally, we recall a result which connects calibrated curves with limiting differentials (see [13] and [33).

Proposition 2.9. Let $x \in \mathbb{T}^{n}$ and $u: \mathbb{T}^{n} \rightarrow \mathbb{R}$ be a viscosity solution of the Hamilton-Jacobi equation (2.1). Then $p \in D^{*} u(x)$ if and only if there exists a $C^{1}$ curve $\gamma:(-\infty, 0] \rightarrow \mathbb{T}^{n}$ with $\gamma(0)=x$ which is $\left(u, L_{c}, \alpha(c)\right)$-calibrated, and $p=\frac{\partial L_{c}}{\partial v}(x, \dot{\gamma}(0))$.

2.4. Barrier functions. For $t>0, x, y \in \mathbb{T}^{n}$ and $c \in \mathbb{R}^{n}$, we introduce the following quantity

$$
h_{t}^{c}(x, y)=\inf \int_{0}^{t} L_{c}(\gamma(s), \dot{\gamma}(s)) d s,
$$

where the infimum is computed over all absolutely continuous arcs $\gamma:[0, t] \rightarrow \mathbb{T}^{n}$ such that $\gamma(0)=x$ and $\gamma(t)=y$.

Let $c \in \mathbb{R}^{n}$ and let $h_{t}^{c}(x, y)$ be defined as above. Peierls' barrier is defined as

$$
h_{c}(x, y)=\liminf _{t \rightarrow \infty} h_{t}^{c}(x, y)+\alpha(c) t .
$$

We call $\mathscr{A}_{c}=\left\{x \in \mathbb{T}^{n}: h_{c}(x, x)=0\right\}$ the projected Aubry set. It is well known that $\mathscr{A}_{c}$ is nonempty and compact for any $c \in \mathbb{R}^{n}$.

Proposition 2.10. (23]) If Peierls' barrier $h_{c}$ is finite then, for each $x \in \mathbb{T}^{n}$, $u_{c}(y):=h_{c}(x, y)$ is a global critical viscosity solution of (2.1). Moreover, for any $x, y \in \mathbb{T}^{n}$, there is an arc $\xi:(-\infty, 0] \rightarrow \mathbb{T}^{n}$, with $\xi(0)=y$, such that

$$
u_{c}(\xi(0))-u_{c}(\xi(-t))=\int_{-t}^{0} L_{c}(\xi(s), \dot{\xi}(s)) d t+\alpha(c) t, \quad \forall t \geqslant 0 .
$$


Let $c \in \mathbb{R}^{n}$ and let $h_{c}$ be Peierls' barrier. The barrier function $B_{c}^{*}(x)$ is defined by Mather $(29])$ as

$$
B_{c}^{*}(x)=\inf _{y, z \in \mathscr{M}_{c}}\left\{h_{c}(y, x)+h_{c}(x, z)-h_{c}(y, z)\right\}, \quad x \in \mathbb{T}^{n},
$$

where $\mathscr{M}_{c}$ is the projected Mather set, that is, the projection onto $\mathbb{T}^{n}$ of Mather's set $\tilde{\mathscr{M}}_{c}$. Note that $\mathscr{M}_{c} \subset \mathscr{A}_{c}$ (see, e.g., [5] 28] 29]). By Proposition 2.10 $h_{c}(x, \cdot)$ gives a global viscosity solution of (2.1) and $h_{c}(\cdot, x)$ a global critical solution of (2.1) with the Hamiltonian $\breve{H}(x, p)=H(x,-p)$. Fix $y, z \in \mathscr{M}_{c}$ and, for each $x \in \mathbb{T}^{n}$, let

$$
u_{c, y}^{-}(x)=h_{c}(y, x), \quad u_{c, z}^{+}(x)=-h_{c}(x, z) .
$$

Then

$$
B_{c}^{*}(x)=\inf _{y, z \in \mathscr{M}_{c}}\left\{u_{c, y}^{-}(x)-u_{c, z}^{+}(x)-h_{c}(y, z)\right\} .
$$

For any $x, y \in \mathbb{T}^{n}$, define Mather's pseudometric (see 29]) on $\mathscr{A}_{c}$ by

$$
d_{c}(x, y)=h_{c}(x, y)+h_{c}(y, x) .
$$

Two points $x, y \in \mathscr{A}_{c}$ are said to be in the same Aubry class if $d_{c}(x, y)=0$.

Proposition 2.11. Let $x, y \in \mathscr{A}_{c}$ be distinct points in the same Aubry class. Then $h_{c}(x, \cdot)$ equals $h_{c}(y, \cdot)$ up to a constant. If $x, y \in \mathscr{A}_{c}, x \neq y$, belong to different Aubry classes, then $h_{c}(x, \cdot)-h_{c}(y, \cdot)$ is not constant.

From Proposition 2.11, it follows that each Aubry class $A$ determines-up to constants - a viscosity solution of the form $h_{c}(y, x)$ for any $y \in A$. Now, suppose there exists a finite number of Aubry classes $A_{1}, \ldots, A_{k}$, and denote by $u_{i}, i=$ $1, \ldots, k$, the corresponding uniquely determined viscosity solutions. Each of such solutions is called an elementary weak KAM solution?. It is not hard to show that if there exists a unique Aubry class, then we can represent the barrier function $B_{c}^{*}$ in the form

$$
B_{c}^{*}(x)=u_{c, y}^{-}(x)-u_{c, y}^{+}(x):=u_{c}^{-}(x)-u_{c}^{+}(x) .
$$

In this case, $\left(u_{c}^{-}, u_{c}^{+}\right)$is called a conjugate pair of weak KAM solutions (see 21]).

Recall that $\mathcal{S}_{-}$usually denotes the set of all viscosity solution $u_{c}^{-}$of the HamiltonJacobi equation

$$
H(x, c+D u(x))=\alpha(c) .
$$

Setting $\breve{H}(x, p)=H(x,-p)$, it is clear that $\breve{H}$ is also a Tonelli Hamiltonian. Let us denote by $\mathcal{S}_{+}$the set of all viscosity solutions, $-u_{c}^{+}$, of the corresponding HamiltonJacobi equation. Then $\left(u_{c}^{-}, u_{c}^{+}\right)$is a conjugate pair of weak KAM solutions if $u_{c}^{-}(x)=u_{c}^{+}(x)$ for any $x \in \mathscr{M}_{c}$.

Let us consider a conjugate pair $\left(u_{c}^{-}, u_{c}^{+}\right)$of weak KAM solutions. We denote by $\mathcal{I}\left(u^{-}, u^{+}\right)$, the set

$$
\mathcal{I}\left(u_{c}^{-}, u_{c}^{+}\right)=\left\{x \in \mathbb{T}^{n}: u_{c}^{-}(x)=u_{c}^{+}(x)\right\} .
$$

We have $\mathcal{I}\left(u_{c}^{-}, u_{c}^{+}\right) \supset \mathscr{M}_{c}$. Under the assumption that there exists a unique Aubry class, it is easy to see that $\mathcal{I}\left(u_{c}^{-}, u_{c}^{+}\right)=\mathscr{A}_{c}$. In other words

$$
\mathscr{A}_{c}=\left\{x \in \mathbb{T}^{n}: B_{c}^{*}(x)=0\right\} .
$$

\footnotetext{
${ }^{1}$ The concept of elementary weak KAM solution was introduced by Chong-Qing Cheng in an alternative way in [15], see also 22].
} 
A set $\mathscr{L}$ of Tonelli Lagrangians is said to be generic (in the sense of Mañé) if there exists a residual 2 set $\mathcal{O} \subset C^{2}\left(\mathbb{T}^{n}\right)$ and a Tonelli Lagrangian $L_{0}$ such that each $L \in \mathscr{L}$ has the form

$$
L=L_{0}+V
$$

for some $V \in \mathcal{O}$. A similar notion can be given for a set of Tonelli Hamiltonians.

Examples of generic properties of interest to this paper are the following:

(GC1) there exists a unique Aubry class in $\mathscr{A}_{c}$ for fixed $c$,

and

(GC2) there exists a finite number of Aubry classes in $\mathscr{A}_{c}$ for all $c$.

Indeed, a well-known result by Mañé 27] ensures that (GC1) holds for a generic family of Tonelli Hamiltonians. Consequently, for any fixed $c \in \mathbb{R}^{n}$, there is a unique viscosity solution of the equation associated with any Hamiltonian of such a generic family. It is also known that (GC2) is a generic property (7]). In this case, for all $c \in \mathbb{R}^{n}$, there exists a finite number of elementary weak KAM solutions.

It is well known that $u_{c}^{-}$(resp. $u_{c}^{+}$) is a locally semiconcave (resp. semiconvex) function with linear modulus. Then the barrier function $B_{c}^{*}$ is also a locally seminconcave function with linear modulus, see, e.g., [13, Proposition 2.1.5]. Given any conjugate pair $\left(u_{c}^{-}, u_{c}^{+}\right)$of weak KAM solutions, one can lift the problem to the universal covering space $\mathbb{R}^{n}$ defining

$$
v^{-}(x)=u_{c}^{-}(x)+\langle c, x\rangle, \quad v^{+}(x)=u_{c}^{+}(x)+\langle c, x\rangle, \quad x \in \mathbb{R}^{n} .
$$

Then, under the generic condition (GC1), we have

$$
B_{c}^{*}(x)=v^{-}(x)-v^{+}(x), \quad x \in \mathbb{R}^{n} .
$$

It is worth noting that both $D^{*} v^{-}(x)$ and $D^{*} v^{+}(x)$ are contained in the corresponding energy surface, i.e

$$
\begin{array}{ll}
H(x, p)=\alpha(c), & p \in D^{*} v^{ \pm}(x), \\
H(x, p)<\alpha(c), & p \in D^{\mp} v^{ \pm}(x) \backslash D^{*} v^{ \pm}(x) .
\end{array}
$$

Indeed, the former assertion of (2.10) follows directly from the definition of $D^{*} v^{ \pm}(x)$ and the fact that the equation holds at all points of differentiability. In order to justify the latter, one just need to combine the inclusions

$$
D^{\mp} v^{ \pm}(x) \subset\{p: H(x, p) \leqslant \alpha(c)\}
$$

with the property Ext $D^{\mp} v^{ \pm}(x)=D^{*} v^{ \pm}(x)$ (see Proposition 2.8) and the strict convexity of $H(x, \cdot)$.

\section{CONNECTING ORBITS AND CRITICAL POINTS OF BARRIER FUNCTIONS}

3.1. A criterion on Homoclinic orbits. We call $x \in \mathbb{R}^{n}$ a (generalized) critical point of a locally semiconcave function $u$ if $0 \in D^{+} u(x)$. Moreover, $x$ is called a critical point of saddle type if $0 \in D^{+} u(x)$ and $x$ is not a local minimum or maximum point of $u$.

In [12, we proved the following criterion for the existence of homoclinic orbits with respect to the Aubry set under a certain condition on limiting differentials.

\footnotetext{
${ }^{2}$ Recall that, in a complete metric space, a subset is called residual if it is the intersection of a countable family of dense open subsets.
} 
Proposition 3.1. Let $x \in \Sigma_{B_{c}^{*}}$, and let $B_{c}^{*}(x)=u_{c}^{-}(x)-u_{c}^{+}(x)$ where $\left(u_{c}^{-}, u_{c}^{+}\right)$is a conjugate pair of weak KAM solutions. If

$$
D^{*} u_{c}^{-}(x) \cap D^{*} u_{c}^{+}(x) \neq \varnothing,
$$

then there exists a minimal homoclinic orbit with respect to the Aubry set $\tilde{\mathscr{A}}_{c}$ passing through $x$.

It is clear that condition (3.1) is equivalent to

$$
0 \in D^{*} u_{c}^{-}(x)-D^{*} u_{c}^{+}(x)=D^{*} v^{-}(x)-D^{*} v^{+}(x),
$$

where $v^{ \pm}$is defined in (2.9). Moreover, if (3.2) is satisfied, then $x$ must be a critical point of $B_{c}^{*}$. Notice that the fact that $x$ is a singular point of $B_{c}^{*}$ is inessential here.

Example 1. Let $L(x, v)=\frac{1}{2}|v|^{2}-(1-\cos x)$ be a one-dimensional pendulum system. For $c=0, \mathscr{A}_{0}=\{2 k \pi\}$, and $x_{k}=(2 k+1) \pi, k \in \mathbb{Z}$ are singular points of the unique weak KAM solution $u_{0}^{-}$up to constants. It is clear that $u_{0}^{+}=-u_{0}^{-}$, and the barrier function $B_{0}^{*}=2 u_{0}^{-}$. Since $D^{+} u_{0}^{-}\left(x_{k}\right)=D^{-} u_{0}^{+}\left(x_{k}\right)=[-2,2]$, we have $0 \in D^{*} u_{0}^{-}\left(x_{k}\right)-D^{*} u_{0}^{+}\left(x_{k}\right)$, and the two types of separatrices give the expected homoclinic orbits.

Definition 3.2. A vector $\theta \in \mathbb{R}^{n}$ belongs to the contingent cone (or Bouligand's tangent cone) $T_{S}(x)$ iff there exist sequences $\theta_{i} \in \mathbb{R}^{n}$, converging to $\theta$, and $t_{i} \in \mathbb{R}^{+}$, decreasing to 0 , such that

$$
x+t_{i} \theta_{i} \in S, \quad \forall i \geqslant 1 .
$$

The vector space generated by $T_{S}(x)$ is called the tangent space to $S$ at $x$ and is denoted by $\operatorname{Tan}(x, S)$.

We define the superlevel set of $B_{c}^{*}$ with respect to a given $x \in \mathbb{R}^{n}$ as

$$
\Lambda_{x}^{+}=\left\{y \in \mathbb{R}^{n}: B_{c}^{*}(y) \geqslant B_{c}^{*}(x)\right\} .
$$

The following criterion gives sufficient conditions for (3.2) to hold true.

Theorem 3.3. Let $x \in \mathbb{R}^{n}$ be a critical point of $B_{c}^{*}$. Then we have that

$$
0 \in D^{*} u_{c}^{-}(x)-D^{*} u_{c}^{+}(x)
$$

if any of the following conditions is satisfied:

(a) at least one of the two solutions $v^{-}$and $v^{+}$is differentiable at $x$,

(b) the tangent space to $\Lambda_{x}^{+}$at $x$ is such that

$$
\operatorname{dim}\left(\operatorname{Tan}\left(x, \Lambda_{x}^{+}\right)\right) \geqslant n-1,
$$

(c) $n=2$ and there exists a unit vector $\theta$ such that $\langle p, \theta\rangle \geqslant 0$ for all $p \in D^{+} B_{c}^{*}(x)$,

(d) $n=2$ and $x$ is not an isolated critical point of $B_{c}^{*}$.

Remark 3.4. Notice that, when $n=2$, condition $(c)$ above is satisfied whenever $x$ is not a local maximum point of $B_{c}^{*}$.

Proof. Let $x \in \mathbb{R}^{n}$ and let $0 \in D^{+} u_{c}^{-}(x)-D^{-} u_{c}^{+}(x)=D^{+} v^{-}(x)-D^{-} v^{+}(x)$.

First, suppose condition (a) holds. Without loss of generality, we can assume that $v^{+}$is differentiable at $x$ with $D v^{+}(x)=p^{+}$, i.e., $D v^{+}(x)=\left\{p^{+}\right\}$. Then, $D^{+} B_{c}^{*}(x)=D^{+} v^{-}(x)-D v^{+}(x)$ by the sum rule for the superdifferential of concave functions and $p^{+} \in D^{+} v^{-}(x)$ because $x$ is a critical point of $B_{c}^{*}$. Now,

$$
Z_{x, E}:=\left\{p \in T_{x}^{*} M: H(x, p) \leqslant E\right\}
$$


is a nonempty compact convex set and $\partial Z_{x, E}$ is $C^{2}$ smooth or a singleton, under the energy condition $E=\alpha(c)$. Since $p^{+} \in \partial Z_{x, E}$, we conclude that

$$
p^{+} \in D^{+} v^{-}(x) \cap \partial Z_{x, E}=D^{*} v^{-}(x) \text {. }
$$

So, there exists $p^{-} \in D^{*} v^{-}(x)$ such that $p^{-}=p^{+}$, or $0 \in D^{*} v^{-}(x)-D^{*} v^{+}(x)$.

We now assume condition (b). Then there exist linearly independent unit vectors $\left\{\theta_{x}^{i}\right\}_{i=1}^{n-1} \subset T_{\Lambda_{x}^{+}}(x)$. By the semiconcavity of $B_{c}^{*}$, for every $i=1, \ldots, n-1$ there exists a sequence $\left\{x_{k}^{i}\right\} \subset \Lambda_{x}^{+}$, converging to $x$ as $k \rightarrow \infty$, such that

$$
B_{c}^{*}(x) \leqslant B_{c}^{*}\left(x_{k}^{i}\right) \leqslant B_{c}^{*}(x)+\left\langle p, x_{k}^{i}-x\right\rangle+\frac{C}{2}\left|x_{k}^{i}-x\right|^{2}, \quad \forall p \in D^{+} B_{c}^{*}(x) .
$$

This implies that there exist limiting vectors $\theta^{i}$ of $\left\{\left(x_{k}^{i}-x\right) /\left|x_{k}^{i}-x\right|\right\}$, such that

$$
\left\langle\theta^{i}, p\right\rangle \geqslant 0, \quad \forall p \in D^{+} B_{c}^{*}(x) \quad(i=1, \ldots, n-1) .
$$

Consequently, there exist $\lambda_{i} \in \mathbb{R}(i=1, \ldots, n-1)$ such that

$$
\begin{aligned}
& \left\langle\theta^{i}, p^{-}\right\rangle \geqslant \lambda_{i}, \quad \forall p^{-} \in D^{+} v^{-}(x) \\
& \left\langle\theta^{i}, p^{+}\right\rangle \leqslant \lambda_{i}, \quad \forall p^{+} \in D^{-} v^{+}(x) .
\end{aligned}
$$

Let $\ell$ be the intersection of the hyperplanes $\Pi_{i}=\left\{p:\left\langle\theta^{i}, p\right\rangle=\lambda_{i}\right\}, i=1, \ldots, n-1$, and observe that $\ell$ is a straight line because $\left\{\theta^{i}\right\}_{i=1}^{n-1}$ are linearly independent. Since $0 \in D^{+} B_{c}^{*}(x)$, there exist covectors $p^{-} \in D^{+} v^{-}(x)$ and $p^{+} \in D^{-} v^{+}(x)$ such that $p^{-}=p^{+}$. If $p^{-} \notin D^{*} v^{-}(x)$, then there exist $p_{1}^{-}, p_{2}^{-} \in \operatorname{Ext}\left(\ell \cap D^{+} v^{-}(x)\right)$ such that $p^{-}$is in the interior of the line segment $\left[p_{1}^{-}, p_{2}^{-}\right]$. We also have $\ell \cap \partial Z_{x, E}=$ $\left\{p_{1}^{-}, p_{2}^{-}\right\}$since all the extremal points of $D^{+} v(x)$ are contained in $\partial Z_{x, E}$. Similarly, if $p^{+} \notin D^{*} v^{+}(x)$, then there exist covectors $p_{1}^{+}, p_{2}^{+} \in \ell \cap \operatorname{Ext}\left(D^{-} v^{+}(x)\right)$ such that $\ell \cap \partial Z_{x, E}=\left\{p_{1}^{+}, p_{2}^{+}\right\}$. This implies that $\operatorname{Ext}\left(D^{+} v^{-}(x)\right) \cap \operatorname{Ext}\left(D^{-} v^{+}(x)\right) \neq \varnothing$. So, recalling the equality $\operatorname{Ext}\left(D^{ \pm} v^{\mp}(x)\right)=D^{*} v^{\mp}(x)$ once again (see Proposition 2.8), we conclude that $0 \in D^{*} v^{-}(x)-D^{*} v^{+}(x)$.

Next, observe that condition (c) is just a special case of (b).

Finally, suppose $x$ is not an isolated critical point of $B_{c}^{*}$ as in condition (d). Then there exists a sequence of critical points $y_{j}$ converging to $x$. By the semiconcavity of $B_{c}^{*}$, for any $p \in D^{+} B_{c}^{*}(x)$ and $p_{j} \in D^{+} B_{c}^{*}\left(y_{j}\right)$ we have that

$$
\begin{aligned}
& B_{c}^{*}\left(y_{j}\right) \leqslant B_{c}^{*}(x)+\left\langle p, y_{j}-x\right\rangle+\frac{C}{2}\left|y_{j}-x\right|^{2} \\
& B_{c}^{*}(x) \leqslant B_{c}^{*}\left(y_{j}\right)+\left\langle p_{j}, x-y_{j}\right\rangle+\frac{C}{2}\left|y_{j}-x\right|^{2} .
\end{aligned}
$$

Choosing $p_{j}=0$ for all $j$ since each $y_{j}$ is a critical point of $B_{c}^{*}$, and combining the two inequalities above, we have

$$
0 \leqslant\left\langle p, y_{j}-x\right\rangle+C\left|y_{j}-x\right|^{2}, \quad \forall p \in D^{+} B_{c}^{*}(x) .
$$

This means there exists a unit vector $\theta$ which satisfies condition (c).

A celebrated result in the theory of differential dynamical systems from the sixties is Smale's theorem on transversal homoclinic points which describes, in particular, the complicated dynamical behavior produced by Smale's horseshoe. When the

\footnotetext{
${ }^{3}$ Recall that, given any convex set $C \subset \mathbb{R}^{n}$ and supporting hyperplane $H$, the extremal points of $C \cap H$ are still extremal points of $C$ (see, e.g., [1] Lemma 2.7.1]).
} 
Aubry set $\tilde{\mathscr{A}}_{c}$ is composed of hyperbolic fixed points or periodic orbits, the "nondegenerate" condition (3.3) in Theorem 3.3 is closely linked to how the unstable submanfold $\left\{\left(x, D v^{-}(x)\right): x \in \mathbb{R}^{n}\right\}$ and the stable submanifold $\left\{\left(x, D v^{-}(x)\right): x \in\right.$ $\left.\mathbb{R}^{n}\right\}$ intersect.

In general, it is hard to tell whether a critical point $x$ of $B_{c}^{*}$ is a singular point or a regular one although, by semiconcavity, each local minimum point of $B_{c}^{*}$ must be regular. In the special case when $n=2$ and $B_{c}^{*}$ is of class $C^{2}$ in a neighborhood, $B(x, \varepsilon)$, of an isolated critical point $x$, condition (3.3) yields the following dichotomy:

1) $x$ is a non-degenerate critical point of $B_{c}^{*}$.

In this case, the local unstable submanfold $\left\{\left(x, D v^{-}(x)\right): x \in B(x, \varepsilon)\right\}$ and the local stable submanifold $\left\{\left(x, D v^{-}(x)\right): x \in B(x, \varepsilon)\right\}$ intersect transversally, and it is clear that $\operatorname{dim}\left(\operatorname{Tan}\left(x, \Lambda_{x}^{+}\right)\right)=n$.

2) $x$ is a degenerate critical point of $B_{c}^{*}$ such that $D^{2} B_{c}^{*}(x)$ has exactly one eigenvalue equal to 0 with one-dimensional eigenspace.

In this case, the phenomenon of homoclinic tangency may happen and $x$ can be a cusp point of the level set, with $\operatorname{dim}\left(\operatorname{Tan}\left(x, \Lambda_{x}^{+}\right)\right)=n-1$.

It is interesting to compare this analysis to the result in 31, where some hyperbolic assumption on the limit sets of the homoclinic orbits is required.

3.2. Lasry-Lions regularization. It is clear that, in the case of $\mathbb{T}^{n}$, we can regard $u_{c}^{ \pm}$, as well as $B=B_{c}^{*}=v^{-}-v^{+}$, as $\mathbb{Z}^{n}$-periodic locally semiconcave functions on $\mathbb{R}^{n}$. Now we recall the regularization technique, known as sup/inf convolution, which is due to Lasry and Lions [26]. A detailed formulation of this method in the finite dimensional case can be found in 3 .

For any semiconcave function $u^{-}: \mathbb{R}^{n} \rightarrow \mathbb{R}$, any semiconvex function $u^{+}: \mathbb{R}^{n} \rightarrow$ $\mathbb{R}$, and any $\lambda>0$, we define

$$
\begin{aligned}
& u_{\lambda}^{-}(x)=\sup _{y \in \mathbb{R}^{n}}\left\{u^{-}(y)-\frac{1}{2 \lambda}|x-y|^{2}\right\}, \\
& u_{\lambda}^{+}(x)=\inf _{y \in \mathbb{R}^{n}}\left\{u^{+}(y)+\frac{1}{2 \lambda}|x-y|^{2}\right\} .
\end{aligned}
$$

The following result characterizes the fundamental approximation properties of $u^{ \pm}$ by $u_{\lambda}^{ \pm}$when $\lambda>0$ is small enough. For the reader's convenience, we provide a new proof of such properties below.

Proposition 3.5. Suppose $u^{-}: \mathbb{R}^{n} \rightarrow \mathbb{R}$ is a semiconcave function with constant $C$. Then, for every $0<\lambda \leqslant \lambda_{0}, 0<\lambda_{0}<<1$, the function $u_{\lambda}^{-}$in (3.6) satisfies the following.

(P1) $u_{\lambda}^{-}$is of class $C^{1,1}\left(\mathbb{R}^{n}\right)$.

(P2) As $\lambda \searrow 0, u_{\lambda}^{-}$decreases to $u^{-}$and $D u_{\lambda}^{-} \rightarrow D^{+} u^{-}$in the graph sense.

(P3) $\lim _{\lambda \rightarrow 0} D u_{\lambda}^{-}(x)=p_{x}$, where $p_{x}$ is the element of minimal norm of $D^{+} u^{-}(x)$.

(P4) The functions $u^{-}$and $u_{\lambda}^{-}$have the same critical points and critical values when $\lambda<\min \left\{\lambda_{0}, C^{-1}\right\}$.

(P5) In particular, there exists $0<\lambda_{1} \leqslant \lambda_{0}$ such that $u^{-}$and $u_{\lambda}^{-}$have the same local maximum points when $\lambda \in\left(0, \lambda_{1}\right]$.

Proof. Hereafter, we drop the minus superscript and write simple $u, u_{\lambda}$ instead of $u^{-}, u_{\lambda}^{-}$. It is worth noting that the definition of $u_{\lambda}$ in (3.6) is actually a local one, 
that is the supremum, in fact the maximum, is taken in some ball $B(x, \rho)$, where $\rho$ only depends on $\lambda$ and $x$ in our case (see, e.g., [13, Lemma 3.5.2]).

Properties (P1) and (P2), for $0<\lambda<\lambda_{0}, 0<\lambda_{0}<<1$, can be derived directly from [13] except for the fact that $D u_{\lambda} \rightarrow D^{+} u$ in the graph sense. This last property follows from the fact that the semiconcavity costant of $u_{\lambda}$ is uniform for $\lambda$ sufficiently small.

We proceed to prove (P3). For fixed $x \in \mathbb{R}^{n}$, let

$$
F(y, x)=u(y)-\frac{1}{2 \lambda}|x-y|^{2} \quad(y \in \bar{B}(x, \rho))
$$

and set

$$
\begin{aligned}
M(x) & =\left\{y \in \bar{B}(x, \rho): u_{\lambda}(x)=F(y, x)\right\} \\
Y(x) & =\left\{D_{x} F(y, x): y \in M(x)\right\} .
\end{aligned}
$$

We have that $D^{+} u_{\lambda}(x)=\operatorname{co} Y(x)$, the convex hull of $Y(x)$ (see [13, Theorem 3.4.4]). If $0<\lambda \leqslant \lambda_{0}$ for $\lambda_{0}$ small enough, $u_{\lambda}$ is of class $C^{1,1}$ (see [13, Theorem 3.5.3]). In this case, it is clear that $Y(x)$ is a singleton, and so is $M(x)$. Set $M(x)=\left\{y_{\lambda}\right\}$ and $Y(x)=\left\{v_{\lambda}\right\}$ where $v_{\lambda}=\left(y_{\lambda}-x\right) / \lambda$. We note that $y_{\lambda} \in B(x, \rho)$ for $\lambda$ small enough. Since $F(y, x)$ attains its maximum at $y=y_{\lambda}$, we have that $v_{\lambda} \in D^{+} u\left(y_{\lambda}\right)$. By the semiconcavity of $u$, for any $p \in D^{+} u(x)$, we have

$$
\begin{aligned}
u(x) & \leqslant u\left(y_{\lambda}\right)+\left\langle v_{\lambda}, x-y_{\lambda}\right\rangle+\frac{C}{2}\left|x-y_{\lambda}\right|^{2} \\
& \leqslant u(x)+\left\langle p, y_{\lambda}-x\right\rangle+\left\langle v_{\lambda}, x-y_{\lambda}\right\rangle+C\left|x-y_{\lambda}\right|^{2} .
\end{aligned}
$$

Then,

$$
\left\langle p-v_{\lambda}, v_{\lambda}\right\rangle+\lambda C\left|v_{\lambda}\right|^{2} \geqslant 0, \quad \forall p \in D^{+} u(x) .
$$

In view of (3.8), it is easily checked that $\left\{v_{\lambda}\right\}$ is bounded when $0<\lambda \leqslant \lambda_{0}$. Without loss of generality, we suppose $v_{\lambda_{k}} \rightarrow v_{0}$ as $\lambda_{k} \rightarrow 0$. So, taking the limit in (3.8) yields

$$
\left\langle p, v_{0}\right\rangle \geqslant\left\langle v_{0}, v_{0}\right\rangle, \quad \forall p \in D^{+} u(x) .
$$

In other words, $p_{0}=v_{0}$ is the unique element of minimal norm of $D^{+} u(x)$. Since $p_{0}$ is independent of the choice of $v_{\lambda_{k}}$, we have that $\lim _{\lambda \rightarrow 0} v_{\lambda}=v_{0}$ and so $\lim _{\lambda \rightarrow 0} y_{\lambda}=$ $x$, which completes the proof of (P3).

For the proof of (P4), note that if $x$ is a critical point of $u$, taking $p=0$ in (3.8) we have

$$
(\lambda C-1)\left|v_{\lambda}\right|^{2} \geqslant 0 .
$$

It follows that $v_{\lambda} \equiv 0$ for $0<\lambda<C^{-1}$, which means $x$ is also a critical point of $u_{\lambda}$. In this case, $y_{\lambda} \equiv x$ and $u_{\lambda}(x)=u(x)$ for $0<\lambda<C^{-1}$. Conversely, if $x$ is a critical point of $u_{\lambda}$, then $0=D u_{\lambda}(x)$, i.e., $\frac{y_{\lambda}-x}{\lambda}=v_{\lambda}=0$, which implies $y_{\lambda}=x$, and so, $0 \in D^{+} u(x)$ and $u(x)=u_{\lambda}(x)$.

To prove (P5), we suppose $x_{0}$ is a local maximum point of $u$, i.e., $u\left(x_{0}\right) \geqslant u(x)$, for any $x \in B\left(x_{0}, \varepsilon\right)$. Using (P4), we get

$$
u_{\lambda}\left(x_{0}\right)=u\left(x_{0}\right) \geqslant u(z) \geqslant u(z)-\frac{|z-x|^{2}}{2 \lambda}, \quad z \in B\left(x_{0}, \varepsilon\right) .
$$

When $\lambda>0$ is small enough such that the maximum of $u(\cdot)-\frac{|\cdot-x|^{2}}{2 \lambda}$ is achieved in $B\left(x_{0}, \varepsilon\right)$. this implies $u_{\lambda}\left(x_{0}\right) \geqslant u_{\lambda}(x)$, for all $x \in B\left(x_{0}, \varepsilon\right)$. Conversely, if $x_{0}$ is a 
local maximum point of $u_{\lambda}$, i.e., $u_{\lambda}\left(x_{0}\right) \geqslant u_{\lambda}(x)$, for any $x \in B\left(x_{0}, \varepsilon\right)$. Then, Using (P4) again, we have

$$
u\left(x_{0}\right)=u_{\lambda}\left(x_{0}\right) \geqslant u_{\lambda}(x) \geqslant u(x), \quad x \in B\left(x_{0}, \varepsilon\right) .
$$

3.3. Critical points of barrier functions. Recalling the local semiconcavity of the barrier function $B_{c}^{*}(x)$, let $B_{\lambda}\left(0<\lambda<\lambda_{0}\right.$ small enough) be the corresponding Lasry-Lions regularization of $B_{c}^{*}(x)$ defined in (3.6). Then $B_{\lambda}$ has the same critical points as $B_{c}^{*}(x)$ by $(\mathrm{P} 4)$ in Theorem 3.5 . If $x$ is a critical point of the barrier function $B_{c}^{*}(x)$, then $x$ produces homoclinic orbits with respect to Aubry set $\tilde{\mathscr{A}}_{c}$ under any of the conditions of Theorem 3.3

So, our first aim in this section is to look for critical points of the barrier function $B_{c}^{*}(x)$ outside the Aubry set, which is the set of the global minimizers of $B_{c}^{*}(x)$. For this purpose, we will use topological tools to obtain lower bounds for the number of critical points of $B_{c}^{*}(x)$ outside the projected Aubry set.

Let $M$ be a closed smooth $n$-dimensional manifold of class $C^{1}$, and let $\Phi^{t}$ be a $C^{1}$ flow on $M . \Phi^{t}$ is called a gradient-like flow if there exists a function $G: M \rightarrow \mathbb{R}$ such that, for any $x \in M$, either $G\left(\Phi^{t}(x)\right)<G\left(\Phi^{s}(x)\right)$ for all $0 \leqslant t<s$ or $\Phi^{t}(x)=x$ for all $t \geqslant 0$. Such a function $G$ is called a Lyapunov function. A point $x \in M$ is said to be a rest point of $\Phi^{t}$ if the orbit through $x$ is constant and we shall denote by $\operatorname{Rest}\left(\Phi^{t}\right)$ the set of all rest points of the flow.

The following definition of relative Lusternik-Schnirelmann category is due to 18. Let $X$ be a topological space and $A \subset X$. The relative Lusternik-Schnirelmann category of the pair $(X, A)$, denoted by $\operatorname{Cat}(X, A)$, is the least integer $n \geqslant 1$ such that there exist open sets $U_{0}, U_{1}, \ldots, U_{n}$ in $X$, with $A \subset U_{0}$ and $X \subset \cup_{i} U_{i}$, such that, for all $i \geqslant 1$, the set $U_{i}$ are contractible in $X$ and, for $i=0$, there exists a homotopy of pair $4:\left(U_{0} \times[0,1], A \times[0,1]\right) \rightarrow(X, A)$ with $H_{0}$ the inclusion $U_{0} \hookrightarrow X$ and $H_{1}\left(U_{0}\right) \subset A$. It is clear that

$$
\operatorname{Cat}(X, \varnothing)=\operatorname{Cat}(X)
$$

where $\operatorname{Cat}(X)$ denotes the classical Lusternik-Schnirelmann category of $X$.

It is well known that the main interest of the Lusternik-Schnirelmann category comes from the fact that, for any smooth compact manifold $M$, Cat $(M)$ gives a lower bound for the number of critical points of any smooth function $f$ on $M$.

Analogously, let $N \subset M$ be a compact topological submanifold of dimension $n$ of $M$ such that $N$ has a smooth interior and $\partial N=A \cup_{\partial A} B$ with $A$ and $B$ smooth $(n-1)$-dimensional submanifolds of $M$ such that $A \cap B=\partial A=\partial B$. Let $\Phi^{t}$ be a gradient-like flow on $M$ and let $W$ be the corresponding vector field. Assume that $W$ points out of $N$ on $A$ and inside $N$ on $B$. Denoting by $\operatorname{Rest}_{N}\left(\Phi^{t}\right)=\operatorname{Rest}\left(\Phi^{t}\right) \cap N$, we have the following.

Proposition $3.6\left([18)\right.$. Suppose $N$ and $\Phi^{t}$ are as above, then

$$
\operatorname{Cat}(N, A) \leqslant \operatorname{Rest}_{N}\left(\Phi^{t}\right) .
$$

\footnotetext{
${ }^{4}$ Recall that for any pair of topological spaces $X$ and $Y$, and $A \subset X, B \subset Y,(X, A)$ and $(Y, B)$ are called pairs of spaces. A map of pairs $f:(X, A) \rightarrow(Y, B)$ is just a map $f: X \rightarrow Y$ such that $f(A) \subset B$. Two maps of pairs $f, g:(X, A) \rightarrow(Y, B)$ are homotopic if there is a homotopy $F$ with the additional restriction that $F(A \times[0,1]) \subset B$.
} 
Remark 3.7. It is worth noting that the setting we use here is similar to the index pair $(N, A)$ in the theory of Conley index, where $N$ is an isolating neighborhood and $A$ is the exit set for $N$. The only difference is that we use the complement $M \backslash N$ instead of $N$ for our purpose and in this case, if $\Phi^{t}$ is a gradient flow, then the exit set $A$ is empty.

Now we apply Proposition 3.6 to our case under condition (GC1) which, as we recalled above, holds true for a generic family of Tonelly Hamiltonians.

Theorem 3.8. Let $L$ be a Tonelli Lagrangian on $\mathbb{T}^{n}$ and, for any $c \in \mathbb{R}^{n}$, assume condition (GC1) so that the barrier function takes the form $B_{c}^{*}(x)=u_{c}^{-}(x)-u_{c}^{+}(x)$. Then there exist at least $C a t\left(\mathbb{T}^{n} \backslash U\right)$ critical points of $B_{c}^{*}$ outside $\mathscr{A}_{c}$, where $U \supset \mathscr{A}_{c}$ is any sufficiently small open neighborhood of $\mathscr{A}_{c}$.

Proof. For any $\lambda>0$ small enough, $B_{\lambda}$ is of class $C^{1,1}$ and has the same critical points as $B_{c}^{*}$ by $(\mathrm{P} 4)$ of Proposition 3.5. Thus, it is enough to estimate the number of critical points of $B_{\lambda}$ outside $\mathscr{A}_{c}$.

In order to give a lower bound for the number of critical points of $B_{\lambda}$ outside $\mathscr{A}_{c}$, let us suppose there exists an open set $U \supset \mathscr{A}_{c}$, such that $\mathscr{A}_{c}$ is the unique critical set (minimizers of $B_{\lambda}$ ) of $B_{\lambda}$ in $U$. This assumption can be made without loss of generality for, otherwise, $B_{\lambda}$ would have infinitely many critical points outside $\mathscr{A}_{c}$ and the conclusion would hold a fortiori. More precisely, we can assume that there exists $a_{0}>0$ such that any $a \in\left(0, a_{0}\right]$ is a regular value of $B_{\lambda}$, and take $U=U_{a}=\left\{y: B_{\lambda}(y)<a\right\}$ for some fixed $a \in\left(0, a_{0}\right]$. Observe that $U$ is an isolated invariant set of the gradient flow $\Phi_{\lambda}^{t}(\lambda>0$ small enough) generated by the potential function $B_{\lambda}$, that is, $\Phi_{\lambda}^{t}(x)=x_{\lambda}(t), t \in \mathbb{R}$, where

$$
\dot{x}_{\lambda}(t)=D B_{\lambda}\left(x_{\lambda}(t)\right) \text {. }
$$

We can now apply Proposition 3.6 taking $N=\mathbb{T}^{n} \backslash U$ and $A=\varnothing$ because $\Phi_{\lambda}^{t}$ is a gradient flow and $B_{\lambda}$ is the required Lyapunov function. It follows that

$$
\operatorname{Rest}_{\mathbb{T}^{n} \backslash U}\left(\Phi_{\lambda}^{t}\right) \geqslant \operatorname{Cat}\left(\mathbb{T}^{n} \backslash U, \varnothing\right)=\operatorname{Cat}\left(\mathbb{T}^{n} \backslash U\right) .
$$

Then $u_{\lambda}$ has at least $\operatorname{Cat}\left(\mathbb{T}^{n} \backslash U\right)$ critical points outside $\mathscr{A}_{c}$.

3.4. Homoclinic orbits outside the Aubry set. In this paper, a homoclinic orbit $(\gamma, \dot{\gamma}):(-\infty,+\infty) \rightarrow T \mathbb{T}^{n}$ (with respect to the Aubry set $\tilde{\mathscr{A}}_{c}$ ) is said to be minimal if there exists $t_{0} \in \mathbb{R}$ such that $\gamma$ is both backward calibrated on $\left(-\infty, t_{0}\right]$ and forward calibrated on $\left[t_{0},+\infty\right)$.

It is clear that, when $\gamma:(-\infty,+\infty) \rightarrow \mathbb{T}^{n}$ produces a minimal homoclinic orbit with respect to Aubry set, there exists $t_{0} \in \mathbb{R}$ such that $x_{0}=\gamma\left(t_{0}\right)$ is a critical point of the barrier function $B_{c}^{*}$. On the other hand, for any critical point of $B_{c}^{*}$ outside $\mathscr{A}_{c}$, we cannot conclude whether it determines an expected minimal homoclinic orbit until verifying any conditions in Theorem 3.3 .

Unfortunately, checking the validity of the conditions of Theorem 3.3 may be difficult in arbitrary dimension $n \geqslant 2$ without any any assumption on $\mathscr{A}_{c}$. On the other hand, conditions c) and d) seem easier to handle in dimension two because, recalling Remark 3.4, it suffices to find critical points of the approximating barrier function $B_{\lambda}$ outside $\mathscr{A}_{c}$ which are not local maximum points.

For our purposes, we need the following result by Hofer. Let $u \in C^{1}\left(\mathbb{R}^{n}\right)$ and let $x \in \mathbb{R}^{n}$ be a critical point of $u . x$ is called a critical point of mountain-pass type 
if, for any open neighbourhood $U$ of $x, u^{-1}((-\infty, u(x))) \cap U$ is nonempty and not pathwise connected.

Proposition 3.9. 25. Let $u \in C^{1}\left(\mathbb{R}^{n}\right)$ and assume that $x_{0}, x_{1} \in \mathbb{R}^{n}$ are distinct points. Define

$$
b=\inf _{\gamma \in \Gamma} \sup _{t \in[0,1]} u(\gamma(t))
$$

where $\Gamma$ is the set of all continuous paths $\gamma:[0,1] \rightarrow \mathbb{R}^{n}$ with $\gamma(0)=x_{0}$ and $\gamma(1)=x_{1}$. If

$$
b>\max \left\{u\left(x_{0}\right), u\left(x_{1}\right)\right\},
$$

then there exists at least one critical point, with critical value $b$, which is either a local minimum point or a point of mountain-pass type.

Remark 3.10. Note that, in the above lemma, (3.10) is satisfied if $x_{0}, x_{1} \in \mathbb{R}^{n}$ are distinct isolated local minimum points of $u$. Indeed, taking closed disjoint balls $B_{0}$ and $B_{1}$ centered at $x_{0}$ and $x_{1}$, respectively, let

$$
b_{0}=\min _{x \in \partial B_{0}} u(x)>u\left(x_{0}\right), \quad b_{1}=\min _{x \in \partial B_{1}} u(x)>u\left(x_{1}\right) .
$$

Then, by (3.9), for any $\gamma \in \Gamma$,

$$
\max _{t \in[0,1]} u(\gamma(t)) \geqslant \max \left\{b_{0}, b_{1}\right\} .
$$

It follows that $b \geqslant \max \left\{b_{0}, b_{1}\right\}>\max \left\{u\left(x_{0}\right), u\left(x_{1}\right)\right\}$.

Theorem 3.11. Let $L$ be a Tonelli Lagrangian on $\mathbb{T}^{2}$ and, for any $c \in \mathbb{R}^{n}$, assume condition (GC1) so that $B_{c}^{*}(x)=u_{c}^{-}(x)-u_{c}^{+}(x)$.

If there exists an open neighborhood $U \subset \mathbb{T}^{2}$ of $\mathscr{A}_{c}$ such that $\mathbb{T}^{2} \backslash U$ is noncontractible, then there exists a minimal homoclinic orbit with respect to the Aubry set $\tilde{\mathscr{A}}_{c}$ outside $\tilde{\mathscr{A}}_{c}$. More precisely, there exists a $C^{2}$ curve $\gamma:(-\infty, \infty) \rightarrow \mathbb{T}^{2}$ which is an extremal of the associated Euler-Lagrange equation, such that the $\alpha$-limit and $\omega$-limit sets of $(\gamma, \dot{\gamma})$ belong to $\tilde{\mathscr{A}}_{c}$. Moreover, $\gamma$ is a backward calibrated curve on $(-\infty, 0]$ and a forward calibrated curve on $[0 . \infty)$.

Proof. Let $U$ be any neighborhood of $\mathscr{A}_{c}$. By Theorem 3.8 there exist at least $\operatorname{Cat}\left(\mathbb{T}^{2} \backslash U\right)$ critical points of $B_{c}^{*}$ in $\mathbb{T}^{2} \backslash U$, and we have $\operatorname{Cat}\left(\mathbb{T}^{2} \backslash U\right) \geqslant 2$ since $\mathbb{T}^{2} \backslash U$ is not contractible. Thus, there exist at least two distinct critical points of $B_{\lambda}$ outside $U$, where $B_{\lambda}$ is the Lasry-Lions regularization of $B_{c}^{*}$.

By (P5) in Proposition 3.5 we have that $x$ is a local maximum point of $B_{c}^{*}$ if and only if it is also a local maximum point of $B_{\lambda}$ with $0<\lambda \leqslant \lambda_{1} \leqslant \lambda_{0}$. Now, suppose all the critical points of $B_{\lambda}$ in $\mathbb{T}^{2} \backslash U$ are isolated local maximum points. Otherwise, there would exist a critical point of $B_{\lambda}\left(B_{c}^{*}\right)$ which satisfies condition (c) (see Remark 3.4) or (d) of Theorem 3.3 yielding the existence of the expected homoclinic orbit. Since there exist at least two isolated local maximum points of $B_{\lambda}$, say $x_{0}$ and $x_{1}$, then by defining

$$
b_{\lambda}=\sup _{\gamma \in \Gamma} \inf _{t \in[0,1]} B_{\lambda}(\gamma(t))
$$

as in Proposition 3.9 (here we use $-u$ instead of $u$ ), together with Remark 3.10 we have a third critical point $x_{2}$ with the critical value $b_{\lambda}$ which is local maximum or of mountain-pass type in the sense of Hofer. In the latter case, we have the expected conclusion by condition (c) and Remark 3.4 In the former case, we have 
a third isolated local maximum point of $B_{\lambda}$, say $x_{2}$. Inductively, we can construct a sequence of isolated local maximum point of $B_{\lambda}$ (thus, of $B_{c}^{*}$ ), a subsequence of which should converge to a cluster point $\bar{x}$. This contradicts the assumption that all the critical points of $B_{\lambda}$ in $\mathbb{T}^{2} \backslash U$ are isolated local maximum points and completes the proof.

Finally, we would like to point out that not only does our method apply to construct homoclinic orbits with respect to Aubry sets but could be used to connect orbits between different Aubry classes under condition (GC2), which ensures there exists finitely many Aubry classes in $\mathscr{A}_{c}$ and holds true, once again, for a generic family of Tonelly Hamiltonians. Suppose that, for a given $c \in \mathbb{R}^{n}$, there exist distinct Aubry classes $A_{1}$ and $A_{2}$ in $\mathscr{A}_{c}$ and define the barrier function

$$
B_{1,2}(x)=u_{1}^{-}(x)-u_{2}^{+}(x), \quad x \in \mathbb{T}^{n},
$$

where $u_{1}^{-}$(resp. $u_{2}^{+}$) is an elementary backward (resp. forward) viscosity solution associated with class $A_{1}$ (resp. $A_{2}$ ).

Theorem 3.12. Let $L$ be a Tonelli Lagrangian on $\mathbb{T}^{2}$ and assume condition (GC2). Let $c \in \mathbb{R}^{n}$ and suppose there exists an open neighborhood $U \subset \mathbb{T}^{2}$ of $\mathscr{A}_{c}$ such that $\mathbb{T}^{2} \backslash U$ is non-contractible. Then there must exist a connecting orbit between any pair of distinct Aubry classes such that each orbit passes through a critical point of the associated barrier functions $B_{1,2}$ in $\mathbb{T}^{2} \backslash U$. Moreover, such a critical point is of mountain-pass type or a nonisolated local maximum point.

Proof. Since $u^{-}(x)=h_{c}(y, x)$ for some $y \in \mathscr{M}_{c}$, then for any $x \in \mathbb{T}^{2}$ and $p \in$ $D^{*} u^{-}(x)$ there exists a unique $\left(u^{-}, L_{c}, \alpha(c)\right)$ calibrated $C^{1}$ curve $\gamma:(-\infty, 0]$ such that $\gamma(0)=x$ and $p=\frac{\partial L_{c}}{\partial v}(\gamma(0), \dot{\gamma}(0))$. Denote by $y$ the projection of an $\alpha$-limit point of $(\gamma, \dot{\gamma})$ onto $\mathbb{T}^{n}$. Now, recall Aubry classes are connected sets (see, e.g. [17]) and each of them contains an ergodic component of $\mathscr{M}_{c}$. Hence, if we assume that there are only finitely many Aubry classes, the connected components of $\mathscr{A}_{c}$ are finite and must coincide with the Aubry classes. This implies the $\alpha$-limit set of $\gamma$ belongs to the Aubry class containing $y$.

From this point on, the proof of the existence of connecting orbits between the Aubry classes $A_{1}$ and $A_{2}$ uses the same reasoning of the proof of Theorem 3.11 applied to the barrier function $B_{1,2}$.

Remark 3.13. For the study of the existence of possible genuine heteroclinic orbits connecting two distinct Aubry sets $\mathscr{A}_{c_{1}}$ and $\mathscr{A}_{c_{2}}$ with $\left[c_{1}\right] \neq\left[c_{2}\right]$, we need introduce some other kind of barrier functions. Unlike the homiclinic case, we always need condition (GC2) to ensure the finiteness of the Aubry classes for $\mathscr{A}_{c_{1}}$ and $\mathscr{A}_{c_{2}}$.

Fix $c_{1}$ and $c_{2}$, suppose that there exists $i_{1}$ (resp. $i_{2}$ ) distinct Aubry classes $A_{1,1}, \ldots, A_{1, i_{1}}$ in $\mathscr{A}_{c_{1}}$ (resp. $A_{2,1}, \ldots, A_{2, i_{2}}$ in $\mathscr{A}_{c_{2}}$ ). Denote by $u_{c_{1}, j}^{-}\left(\right.$resp. $\left.u_{c_{2}, k}^{+}\right)$, $j=1, \ldots, i_{1}\left(k=1, \ldots, i_{2}\right)$ the elementary backward (resp. forward) viscosity solutions determined by $A_{1, j}\left(\right.$ resp. $\left.A_{2, k}\right)$. We set

$$
v_{j}^{-}(x)=\left\langle c_{1}, x\right\rangle+u_{c_{1}, j}^{-}(x), \quad v_{k}^{+}(x)=\left\langle c_{2}, x\right\rangle+u_{c_{2}, k}^{+}(x) \quad x \in \mathbb{R}^{n}
$$

Now, define the associated barrier functions

$$
B_{j, k}(x)=v_{j}^{-}(x)-v_{k}^{+}(x), \quad x \in \mathbb{R}^{n},
$$

where $j=1, \ldots, n_{1}$ and $k=1, \ldots, n_{2}$. Recall that, if $x$ is a local minimum point of $B_{j, k}$, then there exists an expected connecting orbit between $A_{1, i}$ and $A_{2, j}$. It 
is worth noting that we cannot ensure the existence of critical points of $B_{j, k}$, in general, because, unlike in the homoclinic case, the barrier function $B_{j, k}$ is the sum of a $\mathbb{T}^{n}$-periodic function with a nonzero linear function. We will study this case in the future.

Remark 3.14. Actually, the result in Theorem 3.11 holds true under certain more general assumptions. More precisely, given any conjugate pair of weak KAM solutions $\left(u^{-}, u^{+}\right)$, define

$$
B(x)=u^{-}(x)-u^{+}(x) .
$$

Then, by the same argument of the proof of Theorem 3.11 on $B_{c}^{*}$, under condition (GC1) one can prove the existence of minimal homoclinic orbits outside the Aubry set. The only difference is that, under condition (GC1) or (GC2), we can even determine an Aubry class as a specific $\alpha$ - or $\omega$-limit sets according to a fixed conjugate pair of elementary weak KAM solutions as explained in the proof of Theorem 3.12 The connecting orbits between distinct Aubry classes provided by such a theorem pass through a critical point of mountain-pass type or an nonisolated local maximum point of the barrier function $B_{1,2}$ outside the Aubry set, unlike the ones in [17, 20] which are constructed by the Mañé set in finite covering spaces.

\section{REFERENCES}

[1] Albano, P.; Cannarsa, P. Propagation of singularities for solutions of nonlinear first order partial differential equations. Arch. Ration. Mech. Anal. 162 (2002), no. 1, 1-23.

[2] Albano, P., Cannarsa, P., Nguyen, K.T. and Sinestrari, C., Singular gradient flow of the distance function and homotopy equivalence, Math. Ann., 356(2013), 23-43.

[3] Attouch, H.; Azé, D., Approximation and regularization of arbitrary functions in Hilbert spaces by the Lasry-Lions method. Ann. Inst. H. Poincaré Anal. Non Linéaire 10(1993), no. 3, 289-312.

[4] Bernard, P., Homoclinic orbits to invariant sets of quasi-integrable exact map. Ergodic Theory Dynam. Systems bf20(2000) 1583-1601.

[5] Bernard, P., Connecting orbits of time dependent Lagrangian systems. Ann. Inst. Fourier (Grenoble) 52(2002), no. 5, 1533-1568.

[6] Bernard, P., Existence of $C^{1,1}$ critical sub-solutions of the Hamilton-Jacobi equation on compact manifolds. Ann. Sci. École Norm. Sup. (4) 40 (2007), no. 3, 445-452.

[7] Bernard, P., Contreras, G., A generic property of families of Lagrangian systems. Ann. of Math. (2)167 (2008), no. 3, 1099-1108.

[8] Bolotin, S., Homoclinic orbits in invariant tori of Hamiltonian systems, in: V.V. Kozlov (Ed.), Dynamical Systems in Classical Mechanics, American Mathematical Society Translation Series 2, Vol. 168, 1995, pp. 21-90.

[9] Bolotin, S., Homoclinic trajectories of invariant sets of Hamiltonian systems, Nonlinear Differential Equation Appl. 4 (3) (1997) 359-389.

[10] Bolotin, S. V.; Rabinowitz, P. H. Minimal heteroclinic geodesics for the $n$-torus. Calc. Var. Partial Differential Equations 9(1999), no. 2, 125-139.

[11] Borwein, J. M.; Vanderwerff, J. D., Convex functions: constructions, characterizations and counterexamples. Encyclopedia of Mathematics and its Applications, 109. Cambridge University Press, Cambridge, 2010.

[12] Cannarsa, P., Cheng, W. and Zhang Q. Propagation of singularities for weak KAM solutions and barrier functions, to appear in Comm. in Math. Phys.

[13] Cannarsa, P., Sinestrari, C., Semiconcave functions, Hamilton-Jacobi equations, and optimal control. Progress in Nonlinear Differential Equations and their Applications, 58, Birkhäuser Boston, Inc., Boston, MA, 2004.

[14] Cannarsa, P., Yu, Y. Singular dynamics for semiconcave functions. J. Eur. Math. Soc. 11(2009), no. 5, 999-1024. 
[15] Cheng, C.-Q., Variational construction of diffusion orbits for positive definite Lagrangians. Proceedings of the International Congress of Mathematicians. Volume III, 1714-1728, Hindustan Book Agency, 2010.

[16] Contreras, G., Figalli, A. and Rifford, L., Generic hyperbolicity of Aubry sets on surfaces, preprint, 2012.

[17] Contreras, G.; Paternain, G. P., Connecting orbits between static classes for generic Lagrangian systems. Topology 41(2002), no. 4, 645-666.

[18] Cornea, O.; Lupton, G.; Oprea, J. and Tanré, D., Lusternik-Schnirelmann category. Mathematical Surveys and Monographs, 103. American Mathematical Society, Providence, RI, 2003.

[19] Cui, X.; Cheng, C.-Q.; Cheng, W. Existence of infinitely many homoclinic orbits to Aubry sets for positive definite Lagrangian systems. J. Differential Equations 214(2005), no. 1, 176-188.

[20] Fathi, A., Orbites hétéroclines et ensemble de Peierls. C. R. Acad. Sci. Paris Sér. I Math. 326 (1998), no. 10, 1213-1216.

[21] Fathi, A., Weak KAM theorem in Lagragian dynamics, to be published by Cambridge University Press.

[22] Fathi, A.; Figalli, A.; Rifford, L. On the Hausdorff dimension of the Mather quotient. Comm. Pure Appl. Math. 62(2009), no. 4, 445-500.

[23] Fathi, A., Siconolfi, A. Existence of $C^{1}$ critical subsolutions of the Hamilton-Jacobi equation, Invent. Math. 155(2004), no. 2, 363-388.

[24] Hiriart-Urruty, J.-B.; Lemaréchal, C. Fundamentals of convex analysis. Grundlehren Text Editions. Springer-Verlag, Berlin, 2001.

[25] Hofer, H., A geometric description of the neighbourhood of a critical point given by the mountain-pass theorem. J. London Math. Soc. (2) 31(1985), no. 3, 566-570.

[26] Lasry, J.-M.; Lions, P.-L., A remark on regularization in Hilbert spaces. Israel J. Math. 55(1986), no. 3, 257-266.

[27] Mañé, R., Generic properties and problems of minimizing measures of Lagrangian systems. Nonlinearity 9(1996), no. 2, 273-310.

[28] Mather, J. N., Action minimizing invariant measures for positive definite Lagrangian systems, Math. Z. 207(1991), no. 2, 169-207.

[29] Mather, J. N., Variational construction of connecting orbits, Ann. Inst. Fourier (Grenoble) 43(1993), no. 5, 1349-1386.

[30] Palis, J.; Takens, F., Hyperbolicity and sensitive chaotic dynamics at homoclinic bifurcations. Cambridge Studies in Advanced Mathematics, 35. Cambridge University Press, Cambridge, 1993.

[31] Pujals, E.R.; Sambarino, M., Homoclinic tangencies and hyperbolicity for surface diffeomorphisms. Ann. of Math. (2) 151(2000), no. 3, 961-1023.

[32] Rabinowitz, P. H.; Tanaka, K. Some results on connecting orbits for a class of Hamiltonian systems. Math. Z. 206(1991), no. 3, 473499.

[33] Rifford, L., On viscosity solutions of certain Hamilton-Jacobi equations: regularity results and generalized Sard's theorems. Comm. Partial Differential Equations 33 (2008), no. 1-3, $517-559$.

[34] Zheng, Y.; Cheng, C.-Q. Homoclinic orbits of positive definite Lagrangian systems. J. Differential Equations 229(2006), no. 1, 297-316.

[35] Zhou, M. Infinity of minimal homoclinic orbits. Nonlinearity 24(2011), no. 3, 931-939.

Dipartimento di Matematica, Università di Roma Tor Vergata, Via della Ricerca Scientifica 1, 00133 Roma, Italy

E-mail address: cannarsa@mat.uniroma2.it

Department of Mathematics, Nanjing University, Nanjing 210093, China

E-mail address: chengwei@nju.edu.cn 